\title{
THREE- AND FOUR-NUCLEON DYNAMICS AT INTERMEDIATE ENERGIES*
}

\author{
G. Khatri, W. Parol, I. Ciepae, K. Bodek, St. Kistryn
}

A. Magiera, D. Rozpędzik, A. Wrońska, J. Zejma

The Marian Smoluchowski Institute of Physics, Jagiellonian University Reymonta 4, 30-059 Kraków, Poland

$$
\text { B. Keos, E. Stephan }
$$

Institute of Physics, University of Silesia, Bankowa 12, 40-007 Katowice, Poland

\section{A. Kozela}

The Henryk Niewodniczański Institute of Nuclear Physics PAN

Radzikowskiego 152, 31-342 Kraków, Poland

N. Kalantar-Nayestanaki, J. Messchendorp

Kernfysisch Versneller Instituut, Groningen, 9747 AA, The Netherlands

\section{MAZUMDAR}

Tata Institute of Fundamental Research, Mumbai 400 005, India

\section{SkWIRA-CHALOT}

Faculty of Physics, University of Warsaw, Hoża 69, 00-681 Warszawa, Poland

(Received August 6, 2013)

An experiment, with unpolarized deuteron beam of $160 \mathrm{MeV}$ impinging on liquid hydrogen and liquid deuterium targets, was carried out using BINA detector at KVI in Groningen, the Netherlands. Data were collected for the purpose of obtaining high precision differential cross sections of break-up channels in $d p$ and $d d$ collisions. The elastic scattering data were also collected alongside for the purpose of cross-section normalization. A brief description of the experiment and the data analysis as well as some preliminary results are presented.

DOI:10.5506/APhysPolBSupp.6.1167

PACS numbers: 21.30.- $\mathrm{x}, 24.70 .+\mathrm{s}, 25.10 .+\mathrm{s}, 13.75 . \mathrm{Cs}$

* Presented at the Symposium on Applied Nuclear Physics and Innovative Technologies, Kraków, Poland, June 3-6, 2013. 


\section{Introduction}

At present, we have realistic potential theories which explain the nucleonnucleon interaction very well. The system composed of three nucleons $(3 N)$ is the simplest non-trivial environment, in which various models of the nucleon-nucleon $(N N)$ interaction can be tested. Three-nucleon system dynamics can be investigated quantitatively by comparing observables calculated with the use of Faddeev equations with results of precise measurements. The observables, for a deuteron breakup in collision with a proton, can be predicted using modern realistic pairwise nucleon-nucleon $(N N)$ interactions, combined with model of $3 N$ forces [1]. Moreover, the two- and three-nucleon interactions can be modelled within the coupled-channel (CC) framework by an explicit treatment of the $\Delta$-isobar [2]. Alternatively, the dynamics is generated by the Chiral Perturbation Theory (ChPT), so far at the next-to-next-to-leading order with all relevant $N N$ and $3 N$ contributions taken into account [3]. The modern theoretical calculations include different pieces of nucleon-nucleon dynamics like the above mentioned threenucleon force but also the long-range Coulomb interaction or relativistic effects. Cross-section observables in the region of medium energies are very sensitive to all these effects which reveal their influence in different regions of the phase space.

\section{Experiment}

The experiment was carried out with BINA detector at the KVI in Groningen, The Netherlands. BINA is a $4 \pi$ detection system designed for few nucleon scattering experiments at intermediate energies. BINA is divided into two main parts, forward Wall $\left(\theta: 9^{\circ}-37^{\circ}\right)$ and backward Ball $\left(\theta: 37^{\circ}-165^{\circ}\right)$. The forward Wall consists of (a) multi-wire proportional chamber (for reconstruction of angles of the scattered charged particles), (b) 12 vertical thin plastic scintillator 'stripes', and (c) 10 horizontal thick plastic scintillator 'slabs'. The plastic stripes and slabs form $\Delta E-E$ telescopes for particle identification. The backward Ball is nearly spherically symmetric, and made up of 150 triangular phoswich detector elements. The Ball, at the same time, plays role of reaction chamber as well as the detector. A detailed information about the detector can be found in [4-7] and in references therein. For the purpose of the experiment described in this work, an unpolarized beam of deuterons with an energy of $160 \mathrm{MeV}$ was provided from AGOR cyclotron and was impinged on liquid hydrogen and liquid deuterium targets. 


\section{Data analysis and results}

Data were collected and a preliminary presorting was performed. Parts of runs characterized with unstable beam current or problems in functioning of any system elements were carefully removed. Geometry cross-check as well as correction of beam-shift from the target center were performed with the help of $d p$ elastic channel, allowing very precise momentum reconstruction of the detected charged particles. The basic assumptions of selection of the elastic scattering events were: (1) Coincidences of two charged particles in the detectors, and (2) Co-planarity $\left(\Delta \phi=180^{\circ}\right)$ of the double-track coincident events. So far, the data analysis is limited to the forward Wall. Preliminary results are shown in figure 1, where the top-left and top-right panels show typical $\Delta E-E$ particle identifications for $d p$ and $d d$ reactions respectively (note: energies are not calibrated and, therefore, axis scales should not be compared), and the bottom panel shows $d p$-breakup kinematics (two protons from $d p \rightarrow p p n$ reaction, the neutron is undetected) for the angular configuration $\theta_{1}=20 \pm 1^{\circ}, \theta_{2}=25 \pm 1^{\circ}$ and $\phi_{2}-\phi_{1}=160 \pm 3^{\circ}$. The elastic and breakup channels in $d p$-reaction are clearly visible in the
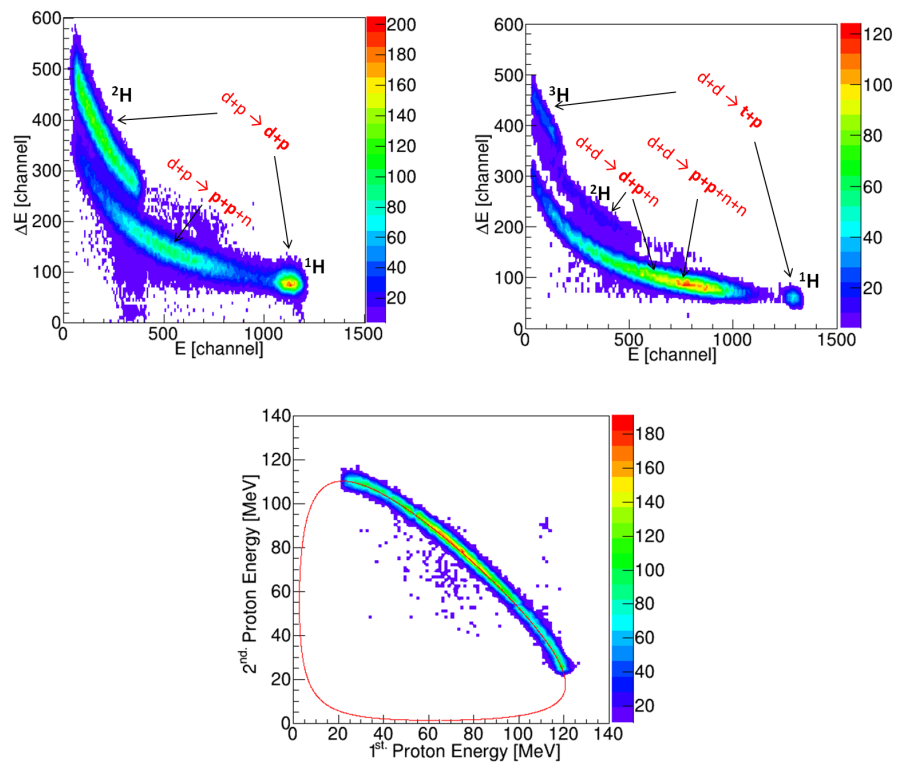

Fig. 1. Preliminary results of $d p$ and $d d$ reactions with the incident deuteron beam energy of $160 \mathrm{MeV}$. The top-left and the top-right panels show typical $\Delta E-E$ plots (without energy calibration) for $d p$ and $d d$ reactions respectively, and the bottom panel shows $d p$-breakup channel fitted with kinematic curve (with energy calibration) for the angular configurations $\theta_{1}=20 \pm 1^{\circ}, \theta_{2}=25 \pm 1^{\circ}$ and $\phi_{2}-\phi_{1}=160 \pm 3^{\circ}$. 
top-left panel. Due to kinematical restrictions (lower detection energy threshold and limited $\theta$ range of Wall), the neutron transfer channel $d d \rightarrow t p$ is relatively less populated and very limited on energy ranges (very low energy tritons and corresponding scattered protons carry the rest of the reaction energy). Moreover, the $d d$-elastic reaction (target and projectile being equal in mass) occurs at $\theta_{2}-\theta_{1}=90^{\circ}$, hence the coincidence of both the elastically scattered deuterons requires also signals from the backward Ball. But since the present analysis refers only to the forward Wall, the $d d$-elastic reaction is not identified here. The two breakup channels from $d d$ reaction, three-body final state $(d d \rightarrow d p n)$ and four-body final state $(d d \rightarrow p p n n)$ are visible in a mixture (long tail of proton branch). Energy calibration of the $E$-slabs was performed with the help of Geant4 simulation and special runs with energy degraders. The obtained $d p$-breakup kinematics (bottom panel) is compared to the calculated kinematics for the same angular configuration.

\section{Conclusion and outlook}

The obtained precise experimental data in a wide phase space region can serve as a valid tool for verification of rigorous theoretical calculations which have been and are being developed. The future studies of the $3 N$ system dynamics in the breakup reaction with BINA detector in Cyclotron Center of Bronowice were proposed. Moreover, the experiment using the deuteron beam of 340,360 and $400 \mathrm{MeV}$ impinging on liquid hydrogen target is performed at the WASA detector (in FZ-Jülich, Germany) with the aim to study the relativistic effects in the three-nucleon system.

We acknowledge support by the Foundation for Polish Science - MPD program, co-financed by the European Union within the European Regional Development Fund, Małopolskie Centrum Przedsiębiorczości — Project "Doctus - Małopolski fundusz stypendialny dla doktorantów", the Polish 2013-2015 science founds as research Project No. 2012/05/E/ST2/02313 and funding from the Jagiellonian University within SET project — the project is co-financed by the European Union.

\section{REFERENCES}

[1] W. Glöckle et al., Phys. Rep. 274, 107 (1996).

[2] A. Deltuva et al., Phys. Rev. C67, 034001 (2003).

[3] E. Epelbaum, Prog. Part. Nucl. Phys. 57, 654 (2006).

[4] St. Kistryn et al., Phys. Rev. C72, 044006 (2005).

[5] St. Kistryn et al., Phys. Lett. B641, 23 (2006).

[6] E. Stephan et al., Phys. Rev. C76, 057001 (2007).

[7] E. Stephan et al., Phys. Rev. C82, 014003 (2010). 